\title{
Potencialización del desarrollo psicológico en adolescentes a través de la representación de su ideal moral en el juego dramatizado
}

\author{
Potentialization of psychological development in adolescents through \\ the representation of their moral ideal in dramatized play
}

Daniel Rosas Alvarez ${ }^{1}$

Claudia Amézquita Rescalvo ${ }^{2}$

\section{RESUMEN}

El presente estudio se configura desde la perspectiva histórico-cultural, teniendo como principales referentes a Bozhovich, Elkonin y Vygotski, quienes caracterizaron a la adolescencia como una edad que presenta como línea de desarrollo general a las relaciones sociales entre coetáneos. Este método psicopedagógico aporta al desarrollo de nuevas cualidades psicológicas como el desarrollo de la autoconciencia, y al establecimiento de objetivos a mediano o largo plazo, construcción de relaciones afectivas entre pares o coetáneos, y al desarrollo de una autovaloración que le permite al adolescente posicionarse en un colectivo de acuerdo a sus intereses y motivos. El objetivo fue favorecer el desarrollo psicológico en adolescentes a través de la representación de su ideal moral en el juego dramatizado. Se utilizó el experimento natural $\mathrm{y}$ formativo como parte de una investigación descriptiva. Participaron 8 adolescentes de edades entre los 12 a 16 años de edad. Se realizaron actividades con los adolescentes durante 25 días, cuatro horas al día. Los primeros y los últimos dos días se realizaron actividades de evaluación. Los días restantes corresponden a actividades propiamente de formación: juego de roles,

\begin{abstract}
The present study is configured from the historical-cultural perspective, having as main references Bozhovich, Elkonin and Vygotski, who characterized adolescence as an age that presents social relations between contemporaries as a general line of development. This psychopedagogical method contributes to the development of new psychological qualities such as the development of self-awareness, and the establishment of medium or long-term goals, the construction of affective relationships between peers or peers, and the development of a self-assessment that allows the adolescent to position themselves in a group according to their interests and motives. The objective was to promote psychological development in adolescents through the representation of their moral ideal in dramatized play. The natural and formative experiment was used as part of a descriptive investigation. Eight adolescents aged 12 to 16 years old participated. Activities were carried out with the adolescents for 25 days, four hours a day. Evaluation activities were carried out on the first and last two days. The remaining days correspond to proper training activities: role play, self-assessment and other activities that
\end{abstract}

\footnotetext{
1 Profesor del Programa de Maestría en Psicología de la FES Zaragoza, UNAM, México. ORCID: https://orcid.org/0000-0003-1523-4689. E-mail: daniel.rosas@zaragoza.unam.mx.

2 Representante de la Jefatura de Gobierno de la Ciudad de México, México. ORCID: https://orcid.org/0000-0003-0895-6288. E-mail: claudia_a_r15@hotmail.com.
} 
autovaloración y otras actividades que demandaban para su solución la participación en colectivo. Los resultados muestran que se favoreció el desarrollo de la comunicación estrecha entre coetáneos, y con ella el establecimiento de relaciones íntimas personales. Se concluye que la representación del ideal moral mediante la actividad de juego dramatizado favorece el posicionamiento de los adolescentes en un colectivo que les permite desarrollar las neoformaciones psicológicas propias de la adolescencia.

Palabras Clave: Autovaloración. Desarrollo de la personalidad. Relaciones sociales.

\section{RESUMO}

O presente estudo configura-se na perspectiva histórico-cultural, tendo como principais referências Bozhovich, Elkonin e Vygotski, que caracterizaram a adolescência como uma idade que apresenta as relações sociais entre os contemporâneos como uma linha geral de desenvolvimento. Este método psicopedagógico contribui para o desenvolvimento de novas qualidades psicológicas, como o desenvolvimento da autoconsciência e o estabelecimento de objetivos de médio ou longo prazo, a construção de relações afetivas entre pares ou pares e o desenvolvimento de uma autoavaliação que permite ao adolescente posicionar-se em um grupo de acordo com seus interesses e motivações. $\mathrm{O}$ objetivo foi promover o desenvolvimento psicológico de adolescentes por meio da representação de seu ideal moral em brincadeiras dramatizadas. $\mathrm{O}$ experimento natural e formativo foi usado como parte de uma investigação descritiva. Participaram oito required collective participation for their solution. The results show that the development of close communication between peers was favored, and with it the establishment of intimate personal relationships. It is concluded that the representation of the moral ideal through dramatized play activity favors the positioning of adolescents in a group that allows them to develop the psychological new formations typical of adolescence.

Keywords: Self-assessment. Personality development. Social relationships.

adolescentes com idades entre 12 e 16 anos. As atividades foram realizadas com os adolescentes durante 25 dias, quatro horas por dia. As atividades de avaliação foram realizadas no primeiro e nos últimos dois dias. Os restantes dias correspondem a atividades de formação próprias: dramatização, autoavaliação e outras atividades que exigiram a participação coletiva para a sua resolução. Os resultados mostram que foi favorecido o desenvolvimento da comunicação próxima entre os pares e, com isso, o estabelecimento de relações pessoais íntimas. Conclui-se que a representação do ideal moral por meio da atividade lúdica dramatizada favorece o posicionamento dos adolescentes em um grupo que lhes permite desenvolver as novas formações psicológicas próprias da adolescência.

Palavras-chave:

Desenvolvimento Relações sociais. da

Autoavaliação. personalidade.

\section{Introducción}

La falta de recursos económicos y las exigencias sociales para ir al ritmo de la vida moderna, unidos a la soledad y a las frecuentes dificultades para establecer relaciones afectivas duraderas y gratificantes dentro de las sociedades capitalistas son algunas de las causas de las principales dificultades psicológicas en la adolescencia (PINTADO et. al., 2010). De 
acuerdo a Balderas (2010), el constructivismo es el enfoque que en las últimas decadas se ha posicionado como principal referente en México para la elaboración de programas y métodos educativos de la educación Secundaria, debido a que, en armonía con el sistema capitalista en el que se ha desarrollado, permite responsabilizar al estudiante como el principal responsable de su aprendizaje, lo cual provoca que a los adolescentes se les dificulte comprender el valor de la comunicación ínitima entre coetaneos para el desarrollo social y personal, es decir, para el desarrollo humano (ENKVIST, 2006). El trabajo colaborativo es consdierado sólo como un medio para desarrollar aprendizajes y no como un estilo de convivencia que aporta de manera significativa en el desarrollo psicológico de las personas durante la adolescencia (DÍAZ; PÉREZ; CHIRIBOGA, 2018; ENVIKS, 2006). El paradigma histórico-cultural, elaborado por Vygotski (2006) desde una psicología que tenía como objetivo aportar en la configuración de una sociedad socialista, es actualmente una alternativa para la elaboración de propuestas alternativas en la educación de adolescentes.

Bozhovich (1976), discípula de Vygotski, señaló la relación existente entre una deficiente comunicación entre los adolescentes con el abandono escolar y su participación en actos delictivos y de indisciplina social (BOZHOVICH, 1976). Lo anterior llevó a Bozhovich a señalar la comunicación en las relaciones interpersonales como actividad fundamental de desarrollo en esta edad, misma que se orienta a satisfacer en el adolescente la necesidad de ocupar una posición (reconocida) en el colectivo.

Para Bozhovich (1976) el proceso de desarrollo moral no se reduce a la simple asimilación de conocimientos morales, sino a brindar las condiciones dentro del colectivo y el carácter de las interacciones del mismo que favorecen la formación en los adolescentes de motivos socialmente valiosos y de sus correspondientes modos de comportamiento. Bozhovich (1976) demostró que la organización de la opinión social es una de las condiciones más importantes para la asimilación de las reglas y de las formas de comportamiento correspondientes. Además mostró que el ideal del hombre (ideal moral), 
adoptado o creado por el adolescente, cuyos rasgos trata de imitar y asimilar, representa un motivo moral constantemente actuante.

A pesar de la relevancia de estos planteamientos, prácticamente no existen estudios empíricos en el idioma castellano elaborados desde la perspectiva histórico-cultural que analicen el papel que ejerce el ideal moral entre coetáneos en el desarrollo del adolescente.

Dentro de los pocos reportes que existen en torno al papel de los ideales morales en la formación de la personalidad desde la perspectiva históricocultural, citamos como ejemplo el de Pérez, Vergel y Rodríguez (2007), quienes al estudiar la influencia de la amistad en la formación de cualidades morales en niños de 9 y 10 años, mediante la presentación de ideales (personajes de distintos cuentos de un libro infantil) que expresaran la solidaridad como cualidad a formar, encontraron que en el cumplimiento de las condiciones necesarias para la formación de cualidades morales como la solidaridad, la a mistad es una condición favorecedora, en tanto se refleja la colaboración entre los amigos como indicador que posibilita mayor rendimiento, motivación y refuerzo de las valoraciones en torno al ideal. Un aspecto que nos interesa destacar del estudio citado, es que, a pesar de los interesantes resultados reportados, el ideal es seleccionado por los investigadores y no por los participantes, como lo implementamos en nuestra investigación.

Considerando la importancia del drama en el desarrollo de las emociones y afectos (VYGOTSKI, 2000; OLMOS, 2011), en la presente investigación se utilizó la representación del ideal moral a través del juego dramatizado para analizar las posibilidades de formación de nuevas cualidades de la personalidad en los adolescentes. A pesar de que el juego dramatizado es la actividad rectora de la edad preescolar (ROSAS; AMÉZQUITA; CONDE, 2014; CONDE; ROSAS; AMÉZQUITA, 2014; ELKONIN, 2003), se ha señalado desde la misma teoría histórico-cultural su potencial para favorecer el desarrollo psicológico en la edad adolescente (OLMOS; MARTÍNEZ, 2013), siempre y cuando se considere como material principal de representación no los roles sociales, sino las narraciones sociales que se configuran a partir de un ideal moral. 


\section{Edad psicológica de adolescencia y neo-formaciones}

En la teoría histórico-cultural la edad psicológica se caracteriza por la inclusión de la persona en una nueva situación social de desarrollo, la cual exige la aparición de una nueva actividad rectora que garantice el surgimiento de nuevas formaciones psicológicas para que dicha edad sea superada con éxito (BOZHOVICH, 1976, 1979). Cada edad psicológica posee una línea general de desarrollo, sea esta práctica operacional o de relaciones sociales (ELKONIN, 1986). En el caso de la adolescencia, las relaciones sociales son la línea general de desarrollo desde la que se configura como actividad rectora la comunicación profunda entre coetáneos, la cual tiene como finalidad reproducir en las relaciones entre adolescentes las relaciones existentes entre los adultos (TOLSTIJ, 1989). La actividad rectora posibilita el surgimiento de neoformaciones, de decir, de nuevas cualidades y procesos psicológicos que no existían previamente (SOLOVIEVA; QUINTANAR, 2014). Algunas de las neoformaciones principales que se espera surjan al final de la adolescencia son: el establecimiento de objetivos propios (SOLOVIEVA; QUINTANAR, 2014), el dominio del mundo interior (VYGOTSKI, 2012), la autoconciencia como conciencia social (TOLSTIJ, 1989; VYGOTSKI, 2006), la estabilidad en la autovaloración y la estabilidad en los ideales (BOZHOVICH, 1976).

\section{Objetivo general}

Favorecer el desarrollo psicológico en adolescentes a través de la representación de su ideal moral en el juego dramatizado.

\section{Objetivos específicos}

- Conocer el impacto que tiene la representación del "ideal moral" en el desarrollo de nuevas formaciones correspondientes a la edad psicológica de la adolescencia. 
- Desarrollar la autovaloración de cada adolescente a partir de la valoración colectiva.

- Facilitar el posicionamiento de los adolescentes en un colectivo mediante actividades de comunicación y expresión entre coetáneos.

- Favorecer el desarrollo emocional de cada adolescente participante.

\section{Preguntas de investigación}

- ¿Qué impacto tiene en los adolescentes el trabajar con el "ideal moral" en el desarrollo de nuevas formaciones correspondientes a la edad psicológica de la adolescencia?

- ¿Cómo se favorece el desarrollo de la autovaloración mediante la representación y valoración del ideal moral?

- ¿Cómo se favorece el posicionamiento del adolescente en un colectivo mediante la representación del ideal moral?

- ¿Cómo se favorece el desarrollo emocional en los adolescentes participantes?

\section{Método}

Se utilizó el experimento natural y formativo (TALIZINA, 2000) como parte de una investigación descriptiva.

\subsection{Participantes}

En este proyecto se incluyeron 8 adolescentes de edades entre los 12 a 16 años de edad. Se realizó la invitación a participar en el estudio a adolescentes de la comunidad de Jardines de San Agustín mediante la impresión y distribución de carteles. Se les informó que la actividad formaba parte de un estudio experimental. Los adolescentes asistían a distintas escuelas secundarias dentro de la localidad.

Se obtuvo el consentimiento por parte de los tutores de los adolescentes para la toma de fotografías y la publicación del estudio. 


\subsection{Escenario}

El estudio se realizó en el Centro de Atención Psicológica y Apoyo Educativo Histórico-Cultural (CAPAEHC), ubicado en el municipio de Chimalhuacán del Estado de México.

\subsection{Instrumentos de valoración e intervención}

- Frases incompletas.

- Historias de vida.

- Representación de ideal moral.

- Actividades de comunicación.

- Composiciones.

- Dinámicas conversacionales.

\subsection{Instrumentos para la recogida de datos}

- Notas de campo.

- Narraciones.

- Fotografías.

- Videos.

\subsection{Procedimiento}

Se realizaron actividades con los adolescentes durante 25 días, cuatro horas al día. Los primeros y los últimos dos días se realizaron actividades de evaluación. Los días restantes corresponden a actividades propiamente de formación. Durante cada día se utilizaban dos horas para la planeación y una hora para la representación dramatizada del ideal moral. Se utilizaba una hora para realizar otro tipo de actividades que favorecieran la comunicación entre pares. Las fases de la actividad principal (representación de ideal moral) se describen a continuación:

- Se les presentó a los adolescentes una clase interactiva sobre el significado de ideal moral, asimismo se brindaron ejemplos de personajes históricos en el ámbito académico, cultural y social; por último se realizó una actividad creativa donde los facilitadores dieron a conocer sus ideales morales a 
manera de ejemplo. Se registró en este primer acercamiento el ideal referido por cada adolescente, permitiendo construir a cada adolescente algún objeto, símbolo o material gráfico que simbolizará su ideal moral, identificando alguna característica esencial de éste (Figura 1). Los ideales morales que expresaron los adolescentes tenían que ver con alcanzar un nivel académico universitario, para poder adquirir así una estabilidad social, económica, y en algunos casos inteligencia; algunos otros tenían que ver con la posibilidad de tener otras oportunidades laborales y lograr establecer relaciones afectivas positivas, ya fuesen de amistad o de noviazgo, configuradas por sentidos de confianza, comprensión y amabilidad con los otros; por último, se identificaron ideales relacionados al deporte que representaban virtudes de constancia, perseverancia, esfuerzo y dedicación para el logro de aquello que algunos tanto buscaban: un reconocimiento social-afectivo. Los ideales expresados en este primer acercamiento fueron la pauta para continuar el trabajo subsecuente.

Figura 1. Clase Interactiva.

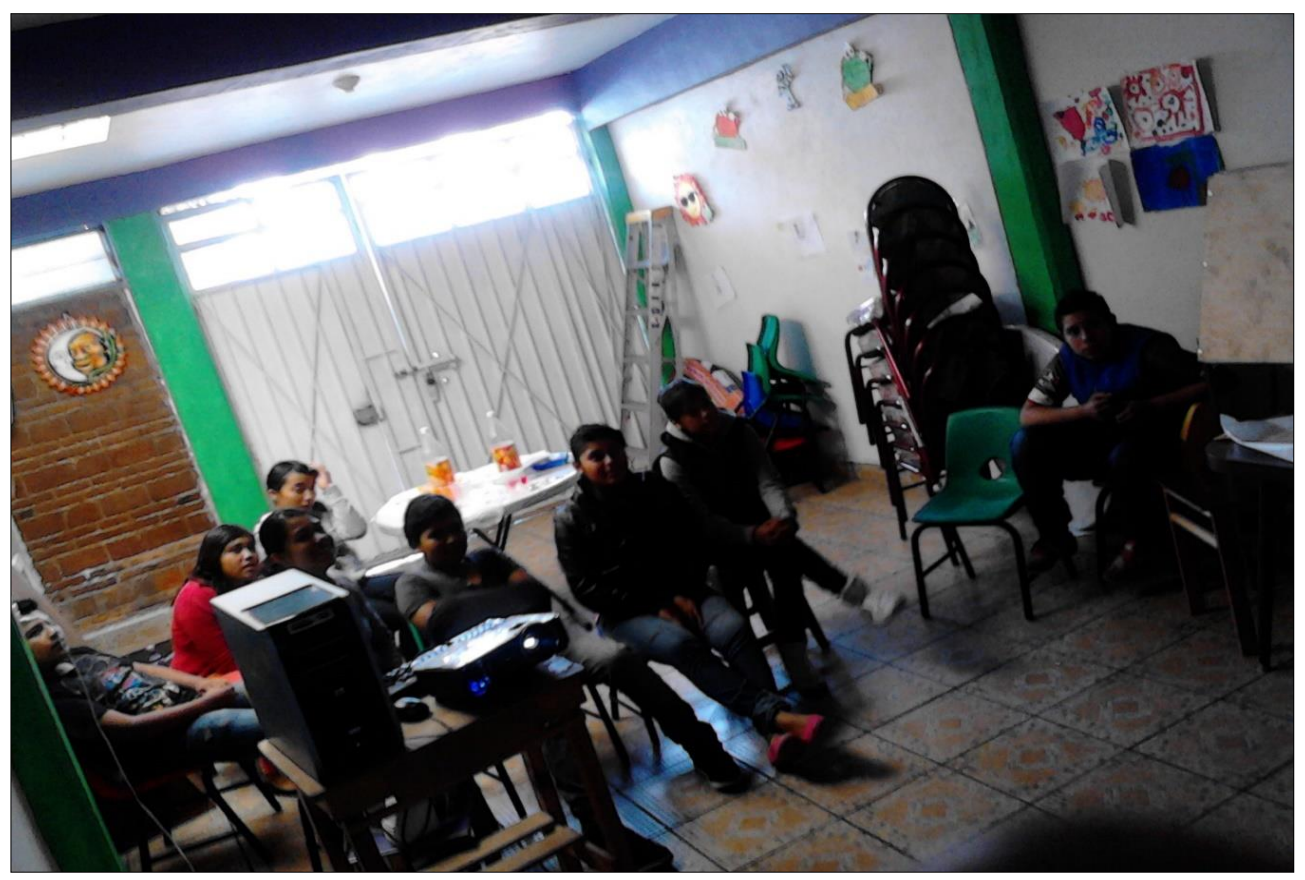

Fuente: archivo personal. 
- Se les solicitó a los estudiantes el completamiento de frases, orientadas a indagar en el conocimiento y jerarquía de sus motivos. Investigadores realizaron dicho análisis.

- Se les solicitó a los estudiantes una composición escrita a cada alumno sobre lo que ellos deseaban ser de grandes. Se les pidió que la compartieran con compañeros e investigadores. (Figura 2).

Figura 2. Composición sobre el ideal moral.

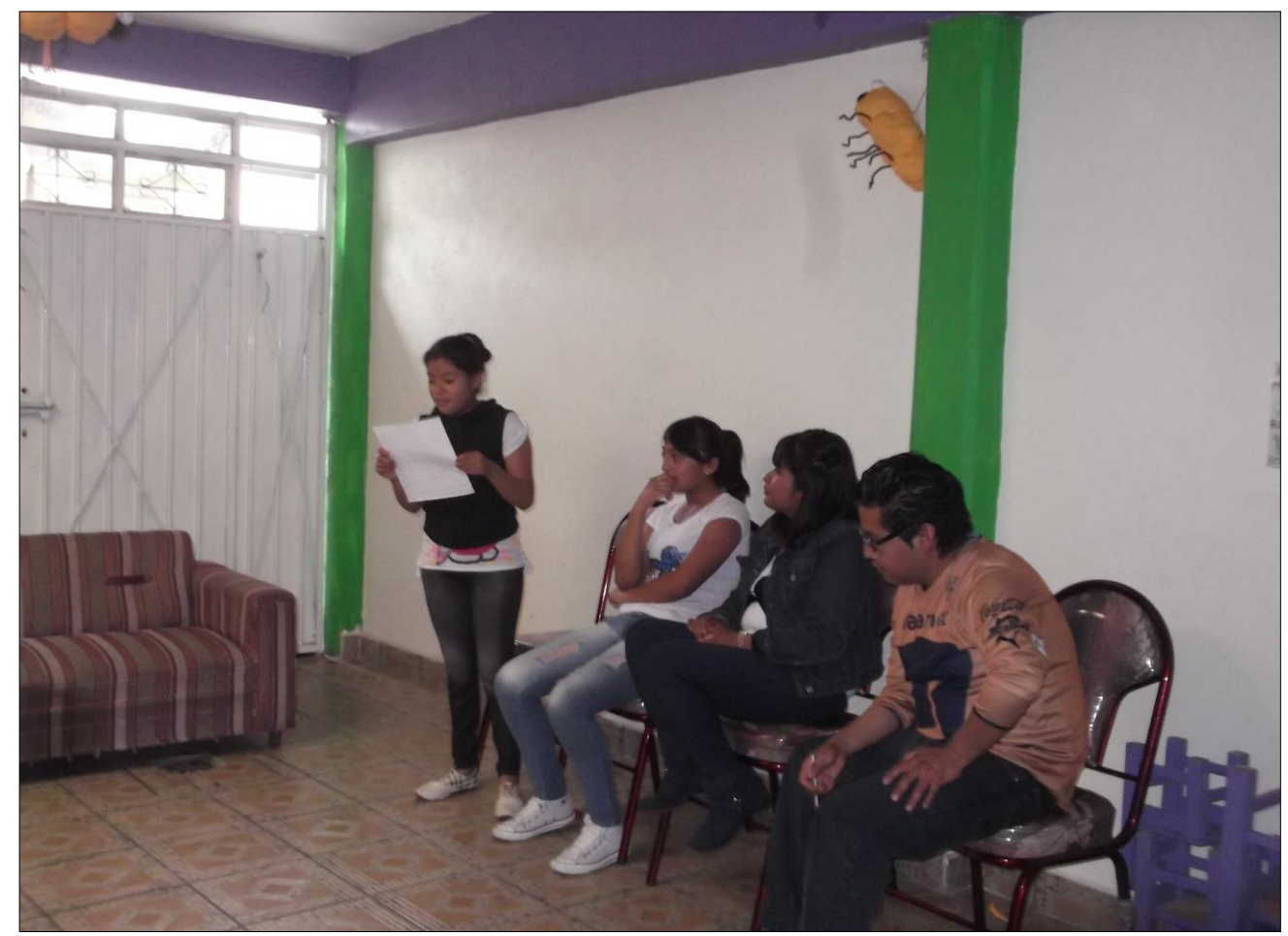

Fuente: archivo personal.

- Se les pidió a los estudiantes que ellos mismos analizaran por escrito qué tanto se correspondía su composición con su ideal moral. Se les solicitó la entrega de dicho análisis.

- Los investigadores realizaron un análisis de contenido de ambos materiales (frases y composición), preparando a partir de dicho análisis dinámicas conversacionales con los adolescentes. Las dinámicas 
conversacionales tuvieron el objetivo de indagar sobre los aspectos más significativos de sus escritos.

- Los investigadores realizaron una narración sobre cada alumno, a partir de lo recopilado en las frases incompletas, composición y dinámicas conversacionales, donde señalaron la jerarquización de motivos de cada adolescente. Concluyendo que la jerarquización de motivos de varios de ellos reflejaban en primer lugar la satisfacción de necesidades afectivas estrechas en su círculo familiar. En otros adolescentes identificamos en primer lugar necesidades relacionadas con el estudio. En otras solamente la oportunidad de incluirse en otros contextos sociales que les permitiesen construir otras oportunidades de un futuro mejor.

- Se les explicó a los adolescentes que cada uno de ellos iba a representar (en escena cuasi-teatral) su ideal moral con la colaboración de su equipo.

- Se les orientó en los elementos que debían considerar para la representación dramatizada de su ideal moral: elección de problema o situación social a representar, reparto de personajes, escenas a representar, preparación de material. Se les enfatizó en que la representación debía girar en torno las cualidades identificadas en su ideal moral. Las cualidades que los adolescentes representaron en algunos de estos juegos dramatizados fue la amabilidad, el buen trato a las personas ante algún servicio brindado, el apoyo entre el equipo de trabajo con el fin de resolver alguna dificultad; también se representó la persistencia, la disciplina y el forjamiento del carácter ante una situación difícil a través de la representación de una pelea de box (se representó este juego debido al gran interés de una de las adolescentes en esta disciplina) (Figura 3 y 4 ). 
Figura 3. Juego dramatizado (enfocado en resaltar cualidades del ideal moral de una de las adolescentes).

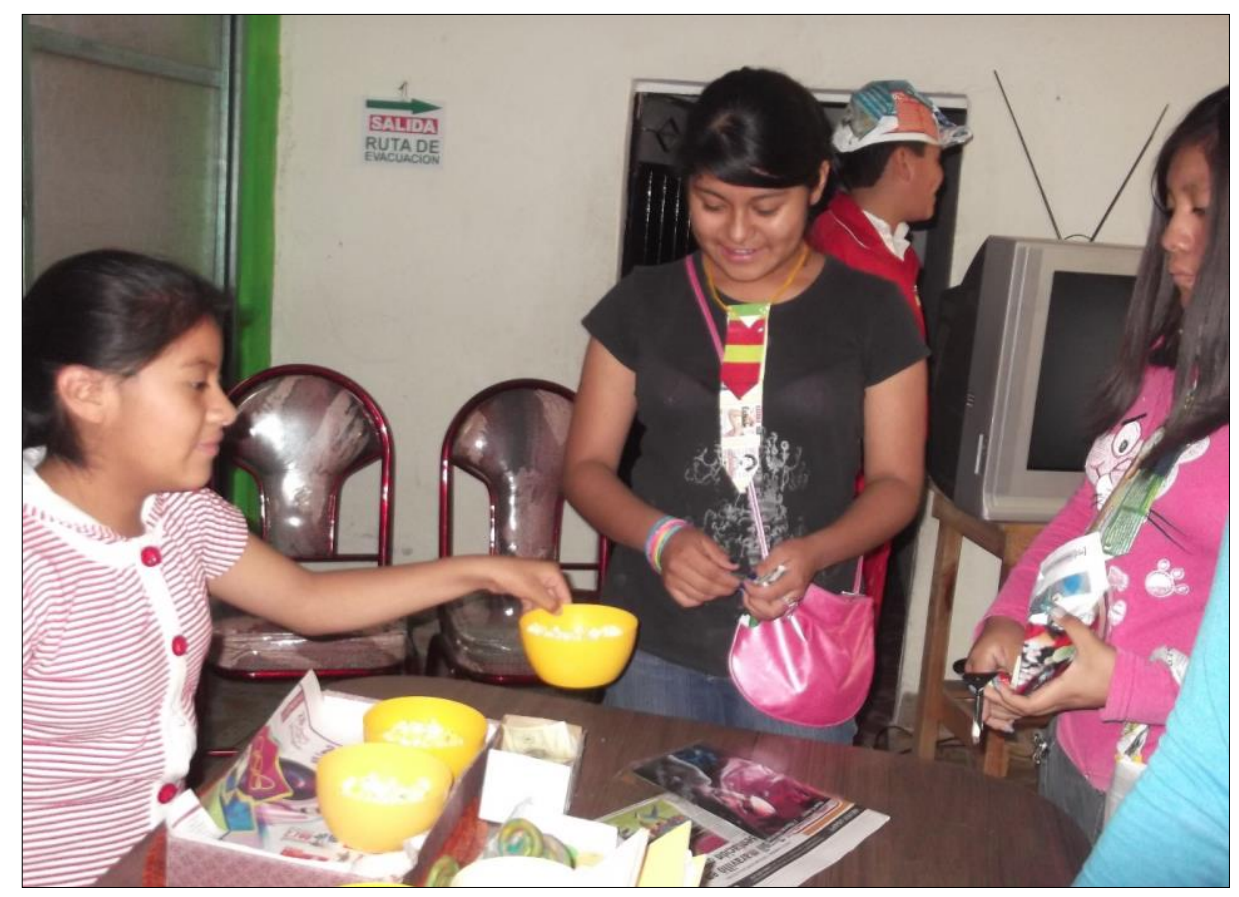

Fuente: archivo personal.

Figura 4. Juego dramatizado (enfocado en resaltar cualidades del ideal moral de una de las adolescentes).

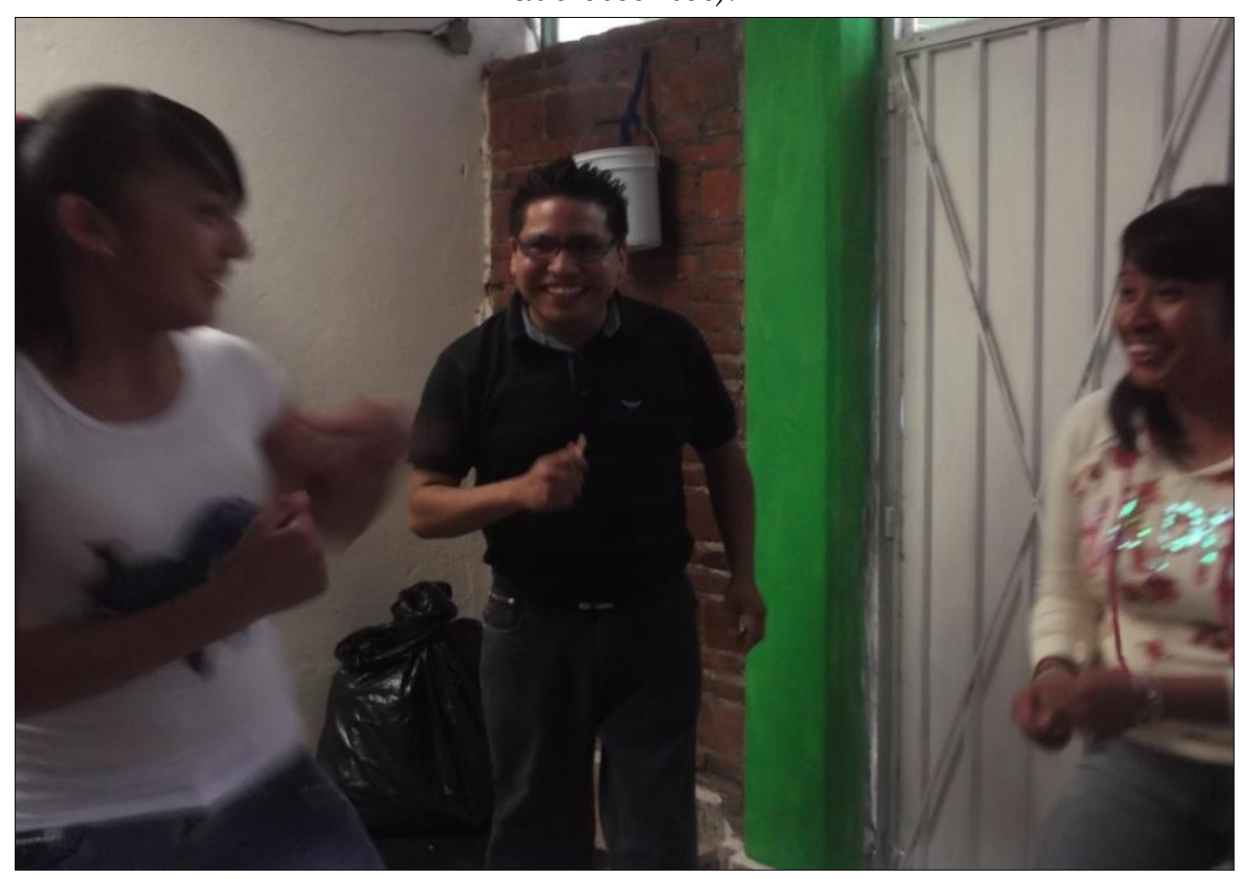

Fuente: archivo personal. 
- Se les solicitó que, posterior a cada representación, realizaran una valoración por escrita, de mínimo cinco renglones, del participante principal (el que ha representado su "ideal moral”). Se les otorgó un esquema que indicaba lo que tenía que contener dicha valoración (descripción de cualidades positivas y negativas).

- El participante principal (que representó su "ideal moral" en determinada sesión) también realizó valoraciones a cada uno del resto de adolescentes.

- Las valoraciones realizadas por todos los adolescentes fueron revisadas antes de ser entregadas a sus destinatarios.

- Se dialogaron en grupo las valoraciones realizadas con el acompañamiento de los investigadores.

- Se les pidió a cada adolescente la elaboración de un escrito de media hoja carta, su sentir respecto a las valoraciones realizadas por sus compañeros.

- A la par de la representación del ideal moral se realizaron otras actividades complementarias orientadas a favorecer la comunicación entre coetáneos.

- Se realizó un grupo de discusión orientado a la reflexión sobre la estructura motivacional de cada adolescente. En él, los adolescentes expresaron la satisfacción obtenida en cada actividad realizada, debido a la oportunidad de compartir intereses con otros y que ayudo a confirmar o construir otras opciones en un futuro cercano con lo que cada uno conoció a través de los otros (Figura 5). 
Figura 5. Grupo de discusión y reflexión.

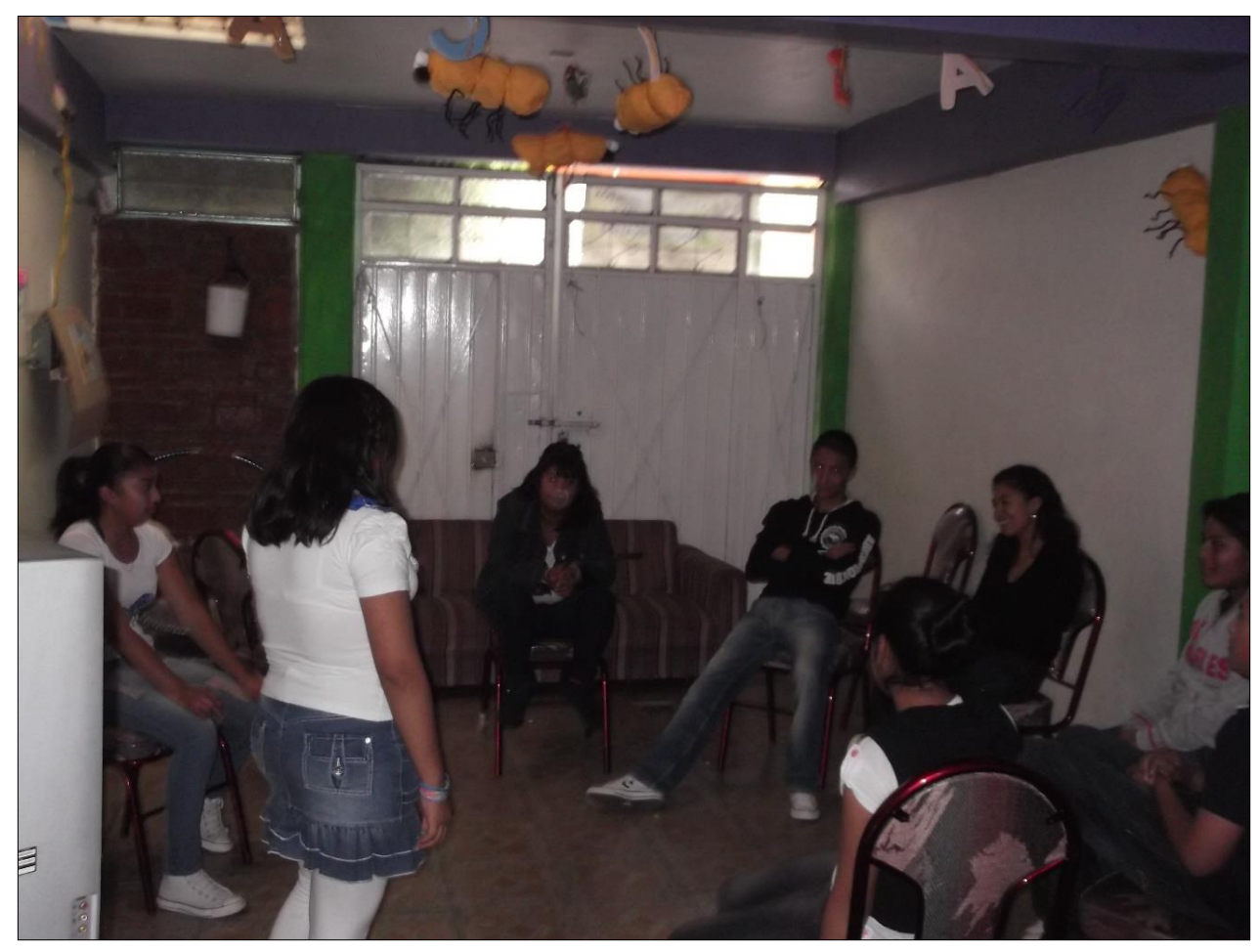

Fuente: archivo personal.

- Al final del curso, se les solicitó a los adolescentes que realizaran una narración sobre su experiencia en las actividades.

\section{Resultados de la evaluación inicial}

Se identificó que los participantes aún no consolidaban algunas cualidades psicológicas de la etapa escolar menor, como es el comportamiento voluntario, capacidad para el aprendizaje individual teórico. A la mayoría de los participantes aún les resultaba difícil autorregularse ante las exigencias sociales. Mostraban también inestabilidad en sus motivos hacía el estudio y no tenían intereses de estudios profesionales. No había indicios de proyectos de vida, ni a largo ni a corto plazo. En el ámbito social se encontró dificultades en la convivencia con sus coetáneos pues algunos de ellos mostraron personalidades con sentidos de timidez y problemas en establecer relaciones de amistad. 


\section{Resultados posteriores a la intervención}

En la tabla 1 se muestran los resultados obtenidos a partir de las actividades formativas, de acuerdo a las distintas áreas de desarrollo intervenidas.

Tabla 1. Resultados de evaluación inicial y final.

\begin{tabular}{|c|c|c|}
\hline $\begin{array}{c}\text { Área de } \\
\text { desarrollo }\end{array}$ & Antes de intervención & Después de intervención \\
\hline $\begin{array}{l}\text { Habilidades de } \\
\text { comunicación }\end{array}$ & $\begin{array}{l}\text { Entre los participantes se tiene a } \\
\text { adolescentes con mayor habilidad } \\
\text { de comunicación interacción } \\
\text { interpersonal, otros a quienes se } \\
\text { les dificulta más pues prefieren } \\
\text { mantenerse aislados o solo } \\
\text { callados observando el escenario } \\
\text { en que se encuentran. }\end{array}$ & $\begin{array}{l}\text { Como resultado de esta } \\
\text { intervención se observó que } \\
\text { aquellos adolescentes que no } \\
\text { contaban con tantas habilidades } \\
\text { de comunicación e interacción } \\
\text { social, pudieron sentirse en esa } \\
\text { libertad de experimentar o de } \\
\text { ser distintos, lo cual impactó en } \\
\text { la formación de nuevas actitudes } \\
\text { en sus relaciones sociales. Se } \\
\text { observó una mejoría en sus } \\
\text { habilidades sociales de } \\
\text { interacción con sus coetáneos y } \\
\text { una mejora de sus relaciones en } \\
\text { otros contextos sociales } \\
\text { (familiares y educativos). }\end{array}$ \\
\hline $\begin{array}{l}\text { Establecimient } \\
\text { o de relaciones } \\
\text { íntimas } \\
\text { personales }\end{array}$ & $\begin{array}{l}\text { No cuentan con amistades estables, } \\
\text { personas que les brinden esa } \\
\text { confianza de poder conversar sobre } \\
\text { lo que les sucede y que impacta su } \\
\text { estado emocional de manera } \\
\text { significativa. } \\
\text { Algo característico entre los } \\
\text { participantes fue que en su mayoría }\end{array}$ & $\begin{array}{l}\text { En varios de los adolescentes se } \\
\text { logró establecer un mayor } \\
\text { vínculo de confianza con sus } \\
\text { coetáneos, algunos de ellos } \\
\text { intercambiaron números } \\
\text { telefónicos, se agregaron en sus } \\
\text { cuentas de redes sociales para } \\
\text { seguir en comunicación. } \\
\text { Encontraron en ellos puntos de }\end{array}$ \\
\hline
\end{tabular}




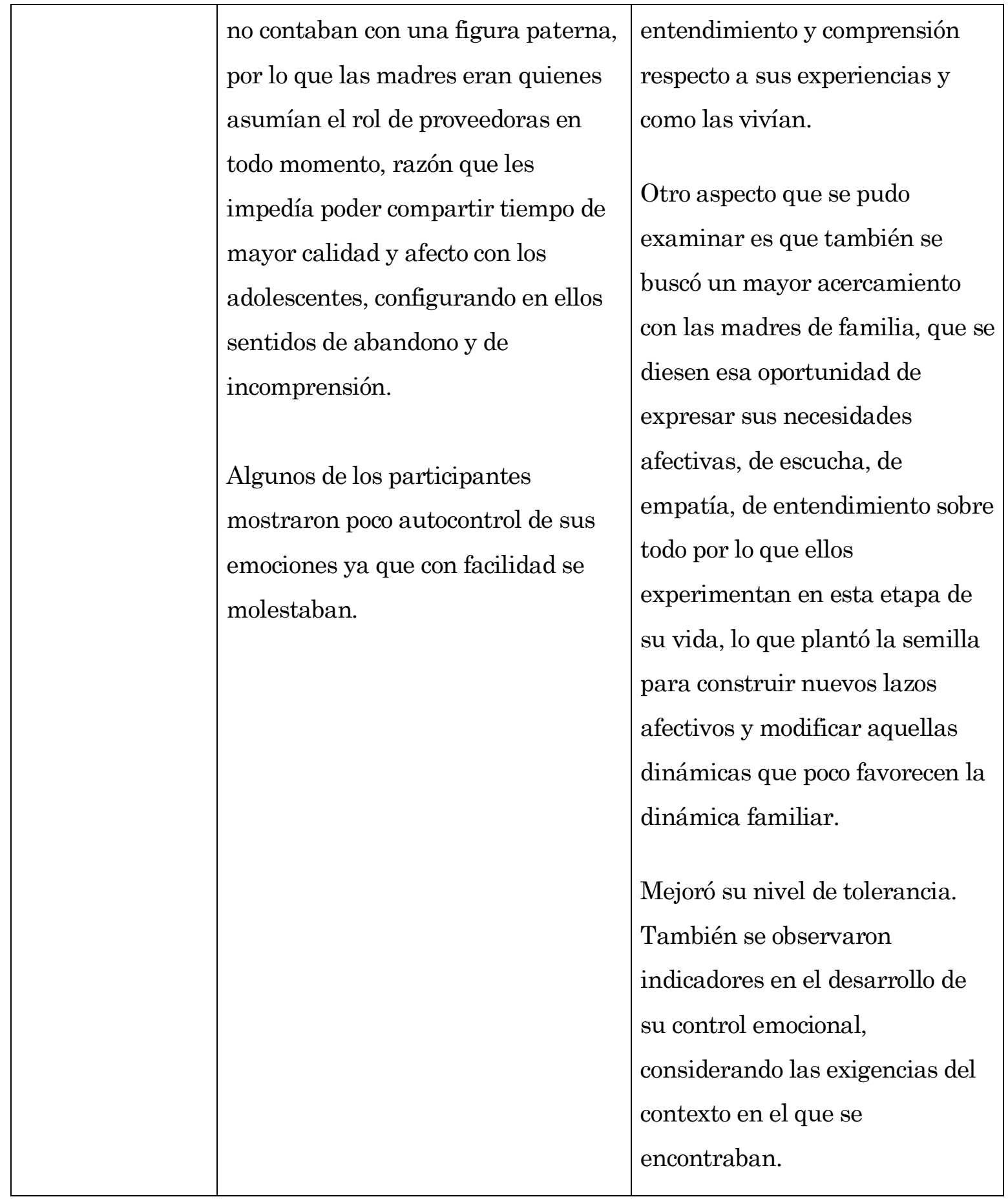




\begin{tabular}{|c|c|c|}
\hline $\begin{array}{l}\text { Comportamien } \\
\text { to voluntario }\end{array}$ & $\begin{array}{l}\text { Se observa entre los adolescentes } \\
\text { que no existe aún una } \\
\text { autorregulación en relación a } \\
\text { reglas sociales, pues tienden a no } \\
\text { esperar turnos, muestran } \\
\text { comportamientos impulsivos, por } \\
\text { lo que, frecuentemente, el } \\
\text { facilitador debe constantemente } \\
\text { regular a los participantes. Lo } \\
\text { anterior impacta negativamente } \\
\text { en su procesamiento cognoscitivo. }\end{array}$ & $\begin{array}{l}\text { Los avances mostrados por los } \\
\text { participantes, evidenciaron } \\
\text { una mayor autorregulación de } \\
\text { su comportamiento, debido a } \\
\text { que entre ellos mismos se } \\
\text { regularon para lograr los } \\
\text { objetivos de las tareas. } \\
\text { Desarrollaron habilidades de } \\
\text { interacción social como una } \\
\text { escucha activa y comprensiva } \\
\text { del otro y la espera de turnos } \\
\text { de participación, lo cual es un } \\
\text { indicador del desarrollo de su } \\
\text { control inhibitorio. }\end{array}$ \\
\hline $\begin{array}{l}\text { Capacidad } \\
\text { para el } \\
\text { aprendizaje } \\
\text { individual } \\
\text { teórico }\end{array}$ & $\begin{array}{l}\text { Entre los adolescentes } \\
\text { participantes nos encontramos con } \\
\text { aquellos que ya tenían esta } \\
\text { cualidad consolidada, mostrando } \\
\text { en todo momento curiosidad por el } \\
\text { conocimiento. También se } \\
\text { detectaron adolescentes que aún } \\
\text { no habían logrado consolidarla, } \\
\text { pues se observaba que ante } \\
\text { actividades que involucraba una } \\
\text { actividad de tipo escolar } \\
\text { mostraban cierto disgusto o } \\
\text { apatía. }\end{array}$ & $\begin{array}{l}\text { Uno de los logros importantes } \\
\text { fue que algunos de los } \\
\text { participantes lograron } \\
\text { encontrar un mayor sentido a } \\
\text { la actividad de estudio, pues al } \\
\text { utilizar sus experiencias como } \\
\text { contenido de la representación, } \\
\text { lograron construir nuevos } \\
\text { sentidos en relación al } \\
\text { aprendizaje. Presentaron } \\
\text { mayor disposición antes las } \\
\text { actividades de mayor } \\
\text { exigencia teórica, y por lo } \\
\text { tanto se permitían mayor } \\
\text { tiempo de dedicación para el } \\
\text { logro de la actividad, ya no se } \\
\text { observaba la apatía } \\
\text { manifestada al inicio. }\end{array}$ \\
\hline
\end{tabular}




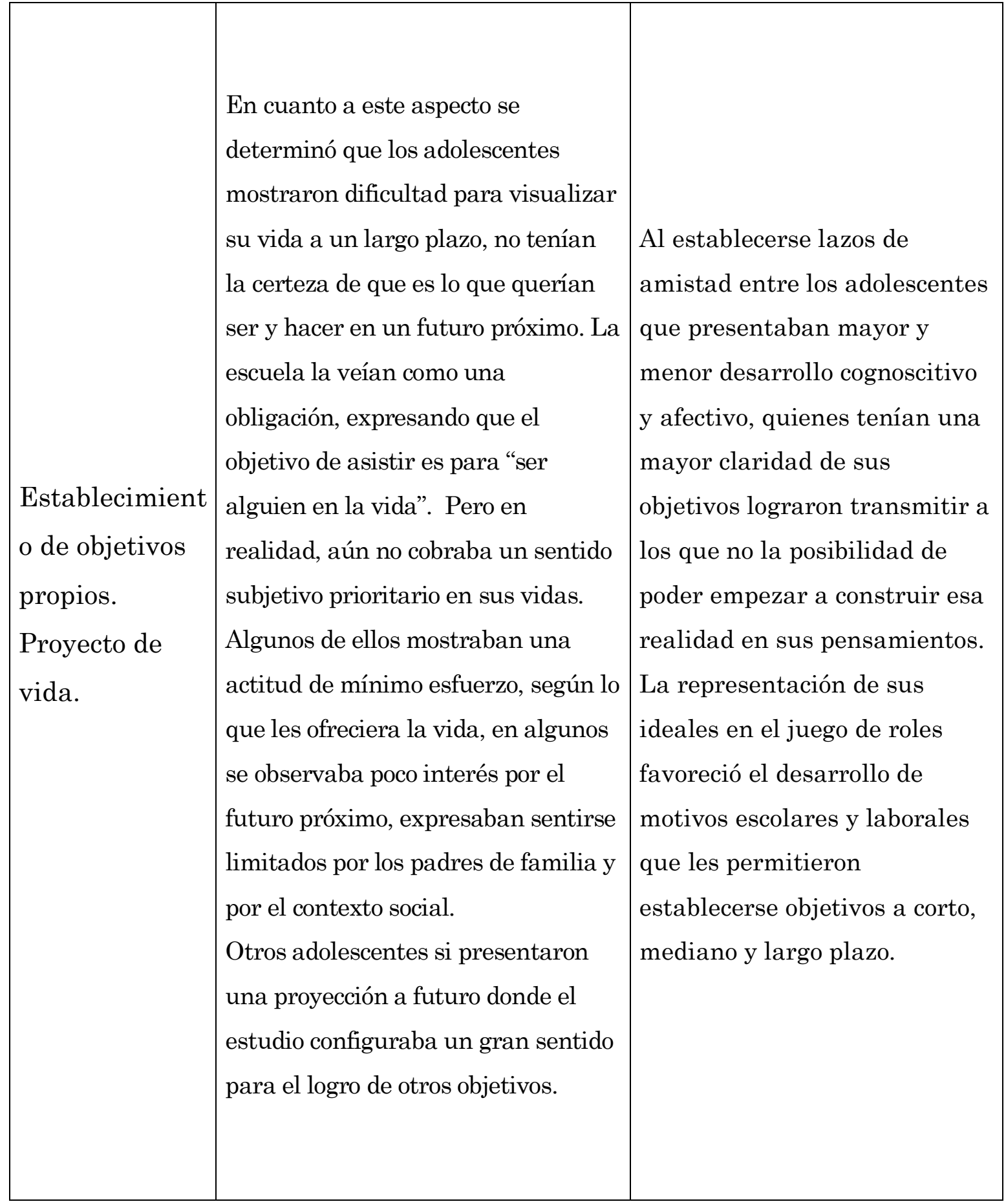

\section{Discusión}

Los resultados muestran que la representación del ideal moral, acompañada de la autovaloración y del trabajo en colectivo, favoreció el desarrollo de la actividad rectora propia de la adolescencia, la comunicación estrecha entre coetáneos, y con ella 
el establecimiento de relaciones íntimas personales (ELKONIN 1986; BOZHOVICH, 1976), lo cual permitió el surgimiento de neo-formaciones específicas de la adolescencia, tales como el establecimiento de objetivos propios (SOLOVIEVA; QUINTANAR, 2005), la autoconciencia (VYGOTSKI, 2006), la asimilación de normas que rigen las relaciones entre personas, el posicionamiento dentro de un colectivo formado por pares, el reconocimiento de compañeros y la sensibilidad a los intereses y exigencias de sus compañeros (TOLTSTIJ, 1989). Además, las actividades realizadas favorecieron la consolidación de algunas formaciones psicológicas, como el comportamiento voluntario y la compasión, que ya deberían de estar consolidadas desde edades previas (SOLOVIEVA; QUINTANAR, 2014). La actividad de representación dramatizada de su ideal moral contribuyó a formar la regulación consciente de sus actos, la consideración en su comportamiento de intereses, deseos y sentimientos de otras personas, cualidades que lamentablemente no son formadas mediante una educación y enseñanza sin emoción que únicamente enseña a los adolescentes a estudiar y a ser obedientes y disciplinados.

Nuestra metodología brinda una alternativa para el trabajo con adolescentes en asignaturas relacionadas con la formación cívica y ética y la orientación vocacional en aulas escolares, ya que a través de ella es posible trabajar la mayoría de las temáticas que las configuran, lo cual favorece que además de garantizar el desarrollo psicológico se pueda cumplir con objetivos escolares.

Una de las limitaciones de nuestra metodología es que, debido a que la formación de motivos estables necesita un proceso largo de consolidación, no es fácil su adaptación para el cumplimiento de objetivos curriculares que demandan resultados inmediatos.

\section{Conclusión}

La metodología propuesta es una alternativa a las metodologías constructivistas que no favorecen la autovaloración y la comunicación íntima entre coetáneos. La representación del ideal moral mediante la actividad de juego dramatizado favorece el posicionamiento de los adolescentes en un colectivo que, al ser fuente importante de desarrollo emocional y cognitivo, les permite desarrollar las neoformaciones psicológicas propias de la adolescencia. 


\section{Referencias}

BALDERAS, G. Las estrategias constructivistas en la enseñanza de la geografía. Tesis de Maestría de la Universidad Veracruzana. México: 2010.

BOZHOVICH, L. El papel de la actividad del niño en la formación de su personalidad. En J. Delval, Lecturas de psicología del niño. El desarrollo cognitivo y afectivo del niño y del adolescente. Madrid: Alianza, 1979.

BOZHOVICH, L. I. La personalidad y su formación en la edad infantil. Cuba: Pueblo y educación, 1976.

CONDE, G.; ROSAS, D.; AMÉZQUITA, C. El juego de roles como medio de prevención del bullying, En PALACIOS, C. Y PINELO, T. Temas de psicología educativa. España: Bubok, 2014.

DÍAZ, E.; PÉREZ, I.; CHIRIBOGA, W. Reflexión sobre el trabajo colaborativo desde sus fundamentos pedagógicos y metodológicos. Revista científico educacional de la provincia Granma. 14 (1), 205-216, 2018.

ELKONIN, D.B. Sobre el problema de la periodización de desarrollo psíquico en la infancia. En: ILIASOV, I.; LIAUDIS, V. (Comp.). Antología de la psicología pedagógica y de las edades. Cuba: La Habana, 1986.

ELKONIN, D. B. Psicología del juego. España: Antonio Machado, 2003.

ENKVIST, I. Repensar la educación. Madrid: Ediciones Internacionales Universitarias, 2006.

OLMOS, A. La teoría dramático-narrativa de Vigotsky en la educación socio emocional. Entre maestros, 11 (38), 20-23, 2011.

OLMOS, A.; MARTÍNEZ, M. Identidad y Narrativa: Recuperando las voces de adolescentes y docentes. México: Facultad de Estudios Superiores Zaragoza, Universidad Nacional Autónoma de México, 2013.

PÉREZ, L. D.; VERGEL, E. A. E.; RODRÍGUEZ, G. C. E. La influencia de la amistad en la formación de cualidades morales en escolares cubanos de 9 y 10 años. Revista Iberoamericana de Educación, No 42/1, 2007. Encontrado en https://rieoei.org/historico/deloslectores/1450Perez.pdf.

PINTADO, M. Y.; JIMÉNEZ, H. Y.; PADILLA, R. M.; GUERRA, C. J. MIGUEL; ANTELO, C. M. E. Trastornos psicológicos en los adolescentes. Una visión general. Rev Psicol. Psiquiatr Nino Adolesc, 9 (1): 11-27, 2010. Encontrado en https://psiquiatriainfantil.org/2010/2010_2.pdf. 
ROSAS, D. AMÉZQUITA, C.; CONDE, G. Una propuesta para la prevención del bullying a través del juego temático de roles. Revista Internacional Magisterio, 69, 56-60, 2014.

SOLOVIEVA, Y.; QUINTANAR, L. Método invariante para la enseñanza de la lectura. Una aproximación histórico-cultural. México: Benemérita Universidad Autónoma de Puebla, 2005.

SOLOVIEVA, Y.; QUINTANAR, L. Enseñanza de la lectura. Método práctico para la formación lectora. México: Trillas, 2014.

TALIZINA, N. Manual de psicología pedagógica. México: Universidad Autónoma de San Luis Potosí., 2000.

TOLSTIJ, A. El hombre y la edad. Moscú: Editorial Progreso, 1989.

VYGOTSKI, L. La imaginación y el arte en la infancia. Madrid: Akal, 2000.

VYGOTSKI, L. Paidología del adolescente. Obras escogidas IV. Madrid: Machado Libros, 2006.

VYGOTSKI, L. Historia del desarrollo de las funciones psíquicas superiores.

Obras escogidas III. Madrid: Machado Libros, 2012. 